\title{
Assessment of low-dose cisplatin as a model of nausea and emesis in beagle dogs, potential for repeated administration
}

\author{
Hannah Kenward · Ludovic Pelligand · Jonathan Elliott
}

Received: 15 February 2014 / Accepted: 10 April 2014 / Published online: 4 May 2014

(C) Springer-Verlag Berlin Heidelberg 2014

\begin{abstract}
Cisplatin is a highly emetogenic cancer chemotherapy agent, which is often used to induce nausea and emesis in animal models. The cytotoxic properties of cisplatin also cause adverse events that negatively impact on animal welfare preventing repeated administration of cisplatin. In this study, we assessed whether a low (subclinical) dose of cisplatin could be utilized as a model of nausea and emesis in the dog while decreasing the severity of adverse events to allow repeated administration. The emetic, nausea-like behavior and potential biomarker response to both the clinical dose $\left(70 \mathrm{mg} / \mathrm{m}^{2}\right)$ and low dose $\left(15 \mathrm{mg} / \mathrm{m}^{2}\right)$ of cisplatin was assessed. Plasma creatinine concentrations and granulocyte counts were used to assess adverse effects on the kidneys and bone marrow, respectively. Nausea-like behavior and emesis was induced by both doses of cisplatin, but the latency to onset was greater in the low-dose group. No significant change in plasma creatinine was detected for either dose groups. Granulocytes were significantly reduced compared with baseline $(P=0.000)$ following the clinical, but not the low-dose cisplatin group. Tolerability of repeated administration was assessed with 4 administrations of an $18 \mathrm{mg} / \mathrm{m}^{2}$ dose cisplatin. Plasma creatinine did not change significantly. Cumulative effects on the granulocytes occurred, they were significantly decreased $(P=0.03)$ from baseline at 3 weeks following
\end{abstract}

H. Kenward $(\bowtie) \cdot$ L. Pelligand $\cdot$ J. Elliott

Department of Comparative Biomedical Sciences, Royal

Veterinary College, Hawkshead Lane, North Mymms, Hatfield,

Herts AL9 7TA, UK

e-mail: hkenward@rvc.ac.uk

\section{Pelligand}

Department of Clinical Sciences and Services, Royal Veterinary College, Hawkshead Lane, North Mymms, Hatfield,

Herts AL9 7TA, UK cisplatin for the 4th administration only. Our results suggest that subclinical doses $\left(15\right.$ and $\left.18 \mathrm{mg} / \mathrm{m}^{2}\right)$ of cisplatin induce nausea-like behavior and emesis but have reduced adverse effects compared with the clinical dose allowing for repeated administration in crossover studies.

Keywords Nausea $\cdot$ Emesis $\cdot$ Cisplatin $\cdot$ Neutropenia $\cdot$ Nephrotoxicity $\cdot$ Arginine vasopressin $\cdot$ Cortisol

\section{Introduction}

The development of a low-dose model of nausea and emesis in the dog has obvious uses within the field of veterinary medicine but also in clinical drug development for human medicine. The dog is close to man in emetic sensitivity (Andrews et al. 1990; Percie du Sert et al. 2012), and a canine model of nausea and validated biomarkers of nausea would have use for preclinical testing of novel anti-nausea compounds and for assessing the emetic liability of novel medicines within the pharmaceutical industry.

Nausea is a subjective experience, which cannot be easily be quantified. Identification of a biomarker would allow for the quantifiable measurement of this subjective sensation. Potential biomarkers of nausea, arginine vasopressin (AVP) and cortisol were identified from the literature. AVP is released in response to fluid loss from vomiting (Koch 1997) and is known to be greatly increased, in man and dogs following cancer chemotherapy agents (Fisher et al. 1982; Cubeddu et al. 1990). Cortisol has been found in humans to be reduced following cisplatin treatment (Morrow et al. 2002), and patients with low urinary cortisol experience significantly higher nausea scores (Fredrikson et al. 1992). 
General consensus is that due to the subjective nature of nausea, it can only be truly studied in humans who are able to verbalize the feeling (Andrews and Sanger 2014). However, behaviors suggestive of nausea and autonomic changes, such as salivation, which accompany nausea in humans, have been identified in animals including the dog (Chen et al. 2003; de la Puente-Redondo et al. 2007; Yu et al. 2009). Due to these limitations of animal models, we will use the term "nausea-like behavior" to describe the experience in the dog.

Cisplatin is a platinum-based cytotoxic drug used in both human and veterinary cancer chemotherapy (Kelland 2007; Barabas et al. 2008). Due to its highly emetogenic properties, cisplatin has been widely utilized as a tool to induce acute nausea and vomiting in preclinical animal models, lasting approximately $6 \mathrm{~h}$ in the dog (de la PuenteRedondo et al. 2007).

Cisplatin acts via both peripheral and central pathways to induce emesis (Minami et al. 2003). The peripheral mechanism of cisplatin is thought to be initiated by 5-HT release in the gastrointestinal system. 5-HT stimulates abdominal vagal afferents, which project to emetic brainstem regions. Sectioning of the dorsal and vagal trunks greatly reduced the emetic response to cisplatin (Andrews et al. 1990). Cisplatin itself or factors released into the bloodstream due to cell damage caused by cisplatin (Cubeddu 1992) act centrally to stimulate the area postrema, also referred to as the 'chemoreceptor trigger zone'. The emetic stimulus is relayed from the area postrema to the 'vomiting center' in the nucleus tractus solitaries, which acts as a central pattern generator to coordinate the emetic reflex (Andrews et al. 1988; Horn 2008). The mechanisms by which cisplatin induces nausea are not well characterized. The subjective nature of nausea and lack of animal models has hindered the identification of these mechanisms.

The clinical dose of cisplatin for the treatment of cancer in dogs is $70 \mathrm{mg} / \mathrm{m}^{2}$ administered every $3-4$ weeks (Ramsey 2012). Cisplatin treatment is associated with numerous adverse effects including nephrotoxicity, myelotoxicity, ototoxicity and neurotoxicity (Barabas et al. 2008). The toxic effects of cisplatin were assessed in healthy dogs; it has a cumulative effect with significant toxicity developing after 4 administrations of cisplatin (Ogilvie et al. 1992).

Development of a model of nausea and emesis utilizing a subclinical dose of cisplatin would fulfill the reduction and refinement objectives of the $3 \mathrm{R}$ (reduction, replacement and refinement) principles of in vivo research (Russell et al. 1959). Model refinement occurs from the identification of a dose, which does not induce the toxicities associated with cisplatin treatment. Refinement of the model to identify a dose that is safe enough for repeated administration will reduce the number of animals required for experimentation as crossover studies, rather than parallel studies, can be carried out where each animal can act as its own control increasing the statistical power of the study. Experiments by Ito et al. (1987) showed that subclinical doses of cisplatin as low as $0.42 \mathrm{mg} / \mathrm{kg}$ equivalent to approximately $8 \mathrm{mg} / \mathrm{m}^{2}$ can induce a response of 3-7 emetic events in the dog. The maximal effect of 11-13 emetic events occurred at $0.85 \mathrm{mg} / \mathrm{kg}$ which is equivalent to $15-18 \mathrm{mg} / \mathrm{m}^{2}$ cisplatin (depending on the weight of the $\mathrm{dog}$ ) and was not increased at higher doses, up to $2.4 \mathrm{mg} / \mathrm{kg}$ (which is equivalent to $43-53 \mathrm{mg} / \mathrm{m}^{2}$ ).

Low doses of cisplatin have been employed for cancer treatment in humans in combination with other anticancer agents. The incidence of nausea and vomiting has been reported in $90 \%$ of patients receiving $15 \mathrm{mg} / \mathrm{m}^{2}$ cisplatin (Samson et al. 1984), prior to the development of modern anti-emetics. More recently, $54 \%$ of patients receiving $4 \mathrm{mg} / \mathrm{m}^{2}$ cisplatin (Ina et al. 2008) and $75 \%$ of patients receiving $10 \mathrm{mg} / \mathrm{m}^{2}$ cisplatin (Imamura et al. 2006) reported nausea and vomiting.

The objectives of the present study were firstly to find a safe repeatable dose of cisplatin which induces significant nausea-like behavior. Secondly the study aimed to investigate the validity of potential "nausea" biomarkers in the dog.

\section{Methods}

Animals

Animal procedures undertaken were approved by the UK Home Office Animals (Scientific Procedures) Act 1986 (ASPA), Project license 70/7269 with ethical approval of the Royal Veterinary College (RVC) Ethics and Welfare Committee. Eight healthy spayed Beagle dogs (Marshall Farms, North Rose, NY, USA), four male and four females weighing from 6.5 to $11.5 \mathrm{~kg}$, aged 1.5 years at the start of the studies, were utilized. Dogs were group housed according to sex on a 12-h light/dark cycle and fed canine Lab diet 5007 (IPS Ltd, London, UK) once daily (100$200 \mathrm{~g}$ adjusted as needed to maintain ideal weight); water was available ad libitum. The dogs were discharged from ASPA and re-homed as pets at the end of the study with the approval of the RVC's Named Veterinary Surgeon.

\section{Experimental design}

\section{Study 1 (clinical versus low-dose cisplatin)}

The clinical dose of cisplatin $\left(70 \mathrm{mg} / \mathrm{m}^{2}\right)$ was compared with a subclinical dose $\left(15 \mathrm{mg} / \mathrm{m}^{2}\right)$, which corresponds to the lower limit of the $0.84 \mathrm{mg} / \mathrm{kg}$ emetic dose identified by Ito et al. (1987). This was a 2-period study blocked by sex 
and by day, a 28-day washout period was observed between the two doses of cisplatin. In period 1, dogs were randomly allocated to treatment with the clinical dose $\left(70 \mathrm{mg} / \mathrm{m}^{2}\right)$ cisplatin (Hospira, Leamington Spa, UK) $(n=4)$ or $0.9 \%$ saline placebo $(n=4)$. In period 2 , dogs that received placebo treatment in period 1 were treated with low-dose $\left(15 \mathrm{mg} / \mathrm{m}^{2}\right)$ cisplatin $(n=4)$ and dogs that received cisplatin treatment in period 1 were treated with $0.9 \%$ saline placebo $(n=4)$.

\section{Study 2 (repeated low-dose cisplatin administration)}

As a part of a separate study, the same 8 dogs from study 1 received repeated administrations of $18 \mathrm{mg} / \mathrm{m}^{2}$, the upper limit of the $0.84 \mathrm{mg} / \mathrm{kg}$ emetic dose identified by Ito et al. (1987). Cisplatin was administered in each of the 4 periods of the study (cumulative dose of $72 \mathrm{mg} / \mathrm{m}^{2}$ ), at least 28-days washout was observed between cisplatin administrations.

\section{Operator safety}

Cisplatin was dispensed with the medical closed system (BD Phaseal, Oxford, UK) under a cytotoxic fume hood. Appropriate personal protective equipment (PPE) was worn by users when handling cisplatin and contaminated waste. Dogs were quarantined for 10 days following cisplatin treatment, PPE was worn to handle dogs, and all waste was considered to be contaminated with cytotoxic material.

\section{Jugular vein catheter placement}

One day prior to cisplatin administration, a double lumen jugular vein catheter (JVC) was implanted under general anesthesia using the modified Seldinger technique (Portillo et al. 2006). Dogs were sedated with medetomidine $(0.01 \mathrm{mg} / \mathrm{kg}$, Medetor, Virbac Ltd, Bury St Edmunds, UK) and butorphanol $(0.2 \mathrm{mg} / \mathrm{kg}$, Dolorex MSD Animal Health, Milton Keynes, UK) both administered by intramuscular injection (i.m). Anesthesia was induced by intravenous administration of propofol (PropoFlo Plus Emulsion for injection, Abbott Animal Health, Maidenhead, UK) to effect and maintained by inhalation of isoflurane (IsoFlo $100 \%$ w/w inhalation vapor, Abbott Animal Health) carried in oxygen. At the end of the procedure, inhalation anesthesia was withdrawn and sedation reversed by atipamezole administered i.m. (0.005 mg/kg; Revertor, Virbac). Analgesia was provided with medetomidine and butorphanol during the transcutaneous catheterization, but due to the noninvasive nature of the procedure, analgesia was not repeated on recovery.

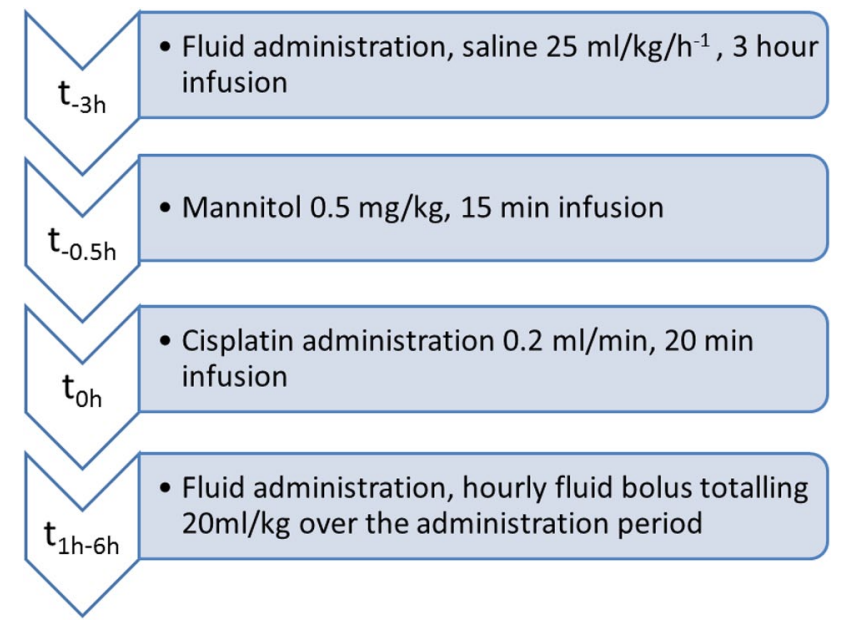

Fig. 1 Fluid and cisplatin administration protocol common to study 1 (high- vs. low-dose cisplatin) and study 2 (4-period repeated lowdose cisplatin administration)

Fluid treatment

The time of initiation of cisplatin infusion was measured in hours and defined as $\mathrm{t}_{0 \mathrm{~h}}$ (Fig. 1). For both study 1 and 2, all dogs were given $0.9 \%$ saline and mannitol infusions to reduce the nephrotoxic effects of cisplatin (Ogilvie et al. 1988) (Fig. 1). A solution of $0.9 \%$ saline (Baxter, Newbury, UK) was infused at $25 \mathrm{ml} / \mathrm{kg} / \mathrm{h}^{-1}$ for $3 \mathrm{~h}$ (from time $t_{-3 \mathrm{~h}}$ to $t_{0 \mathrm{~h}}$ ) via the first lumen of the JVC prior to cisplatin administration. Mannitol (0.5 mg/kg i.v.; Baxter, Newbury, UK) was infused through a $0.22-\mu \mathrm{m}$ filter over a $15-\mathrm{min}$ period from $t_{-0.5 \mathrm{~h}}$ prior to the start of cisplatin administration. After cisplatin administration, a total volume of $0.9 \%$ saline equal to $20 \mathrm{ml} / \mathrm{kg}$ was administered as i.v. boluses via the JVC at hourly intervals over $6 \mathrm{~h}$ following cisplatin treatment.

\section{Cisplatin administration}

Study 1: At $\mathrm{t}_{0 \mathrm{~h}}$, dogs received treatment with $70,15 \mathrm{mg} /$ $\mathrm{m}^{2}$ or $0.9 \%$ saline placebo (Fig. 1). Dogs were weighed on the day preceding the injection. For both doses of cisplatin, the appropriate volume of $1 \mathrm{mg} / \mathrm{ml}$ cisplatin solution for each dog was diluted in $0.9 \%$ saline to produce a standard volume of $40 \mathrm{ml}$. The diluted cisplatin solution was administered through the second lumen of the JVC using automatic dispensing syringe at a flow rate of $2.0 \mathrm{ml} / \mathrm{min}^{-1}$.

Study 2: Cisplatin was repeatedly administered intravenously at the dose of $18 \mathrm{mg} / \mathrm{m}^{2}$ to all dogs as described above during each of the 4 periods of the study. A washout time of 28 days was observed between periods. 
Behavioral assessment

Prior to the commencement of studies, an acclimatization period of 1 month was allowed for the dogs to become familiar with the experimental context. During this period, the observer became accustomed to the dogs normal behavior, which was used as a baseline reference for behavioral observations carried out in study 1 . During the 28-day washout interval between the periods of the study, the dogs were placed in the experimental context weekly to prevent the development of a conditioned response.

Observations of behaviors suggestive of a nausealike state and the number of vomits were recorded for 6-7 $\mathrm{h}$ following cisplatin treatment for study 1 . Nausea was recorded by a single trained observer using a visual analogue scale (VAS) on a line from 0 to $100 \mathrm{~mm}$ (de la Puente-Redondo et al. 2007). The observer makes a judgment on the severity of the dog's nausea-like behaviors based on the presence and frequency of one or more of the following during a 15-min time bin; salivation, lip licking, lethargy, restlessness or turning/circling behavior signaling that vomiting is imminent. A score of $0 \mathrm{~mm}$ indicates 'No "Nausea" and a score of $100 \mathrm{~mm}$ indicates "Worst possible "Nausea". The observer was blinded to treatment allocation.

\section{Blood sampling}

Venous blood samples were collected from the jugular vein catheter at $t=-24$ (baseline), 0, 0.75, 1, 1.5, 2, 2.5, 3, 3.5, 4, 5, 6, 7 (for low dose only) and $24 \mathrm{~h}$ following cisplatin administration. Approximately $5 \mathrm{ml}$ of blood was taken at each time point and collected into EDTA-coated tubes or lithium-heparin-coated tubes. In agreement with ASPA regulations, a maximum of $15 \%$ blood volume was withdrawn in any 28-day period, with no more the $10 \%$ withdrawn in any 24-h period. Blood samples were placed on ice for a maximum of $10 \mathrm{~min}$ before centrifugation at $4,000 \mathrm{~g}$ for $15 \mathrm{~min}$. Plasma samples were aliquoted out into individual tubes; $15 \mu \mathrm{M}$ of protease inhibitor cocktail (SigmaAldrich, UK) was added to tubes containing plasma for vasopressin assay. All samples were snap-frozen on dry ice then stored at $-80{ }^{\circ} \mathrm{C}$.

Potential biomarker measurement

\section{Arginine vasopressin}

Vasopressin was extracted from the plasma, and the concentrations were measured by radioimmunoassay (RIA) (RB-319, Euro-Diagnostica AB, Malmö, Sweden), as per kit instructions. This RIA has been previously validated for use with canine plasma (Tidholm et al. 2005).
Cortisol

Plasma cortisol concentrations were measured by RIA (Coat-a-Count $^{\circledR}$, Siemens, Los Angeles, CA, USA), as per kit instructions, previously validated for use with canine plasma (Olsson et al. 2003).

\section{Plasma creatinine measurement}

Plasma creatinine was measured as a biomarker of acute kidney injury. Blood was collected by cephalic venepuncture into lithium-heparin-coated tubes for dogs treated with cisplatin. A baseline sample was collected the day prior to cisplatin administration and further samples at weeks 1, 2 (study 1 only) and 4 following cisplatin. Samples were spun, and plasma was stored at $-20{ }^{\circ} \mathrm{C}$ until analysis. Plasma creatinine was measured by IDEXX Laboratories (Wetherby, UK) using a photometric method (Jaffe reaction) (AU2700, Beckman Coulter, High Wycombe, UK).

\section{Granulocyte counts}

Whole blood was collected as above on the day prior (baseline) to cisplatin administration and at weeks 1, 2, 3 and 4 following cisplatin. Granulocytes were counted using the animal blood cell counter calibrated for dog blood (Scil Animal Care co., Viernheim, Germany). Dogs with granulocyte counts below $2.0 \times 10^{3} / \mathrm{mm}^{3}$ were considered to be neutropenic and received systemic antibiotic treatment to avoid septic complications (Vail 2009).

Statistical analysis

All statistical analyses were carried out using PASW statistics 18 v 18.0.0 (SPSS: An IBM company, Chicago, IL, USA). All data are presented as mean \pm SEM, and significance levels were set at $P<0.05$.

Study 1: Timecourses of nausea-like behavior and change in potential biomarkers were analyzed using a linear mixed-effect model with first-order autoregressive covariance structure. The statistical model included the fixed effects of treatment group, time and group $\times$ time interaction, the repeated effects of time and the random effect of subject. A least significant difference (LSD) post hoc pairwise comparison of placebo versus treatment was made at each time point if there was a significant interaction between treatment and time.

The area under the curve (AUC) was calculated for timecourse of change in nausea-like behavior and potential biomarkers by determining the trapezoidal area under the curve. AUCs from different dose groups and the difference in the number of vomits were compared with an unpaired two-tailed $t$ test. 
Table 1 Summary of the emetic and nausea responses following cisplatin administration

\begin{tabular}{|c|c|c|c|c|c|c|c|}
\hline \multirow[t]{2}{*}{ Dose } & \multicolumn{3}{|l|}{ Vomiting } & \multicolumn{4}{|c|}{ Nausea-like behaviors } \\
\hline & $\begin{array}{l}\text { Onset time } \\
\text { (h) }\end{array}$ & $\begin{array}{l}\text { Duration } \\
\text { (h) }\end{array}$ & $\begin{array}{l}\text { Mean number } \\
\text { of vomits [range] }\end{array}$ & $\begin{array}{l}\text { Onset time } \\
\text { (h) }\end{array}$ & $\begin{array}{l}\text { Duration } \\
\text { (h) }\end{array}$ & $\begin{array}{l}\text { Peak time } \\
\text { (h) }\end{array}$ & $\begin{array}{l}\text { Peak VAS } \\
(\text { mean } \pm \text { SEM) }\end{array}$ \\
\hline $70 \mathrm{mg} / \mathrm{m}^{2}$ Period 1 & 1.5 & 2.5 & $18[8-32]$ & 1.75 & 2.75 & 3.5 & $72 \pm 16$ \\
\hline $15 \mathrm{mg} / \mathrm{m}^{2}$ Period 2 & 3.75 & 2 & $3.75[0-6]$ & 3.75 & 2.25 & 5.25 & $48 \pm 4$ \\
\hline
\end{tabular}

Nausea was recorded by a trained observer using a visual analogue scale (VAS) on a line from 0 to $100 \mathrm{~mm}$ based on the following nausea behaviors; salivation, lip licking, lethargy and restlessness; onset of vomiting and nausea-like behaviors estimated from visual inspection of the data

A univariate general linear model with LSD post hoc comparison was used to compare relative change from baseline and differences between the two cisplatin treatment groups for plasma creatinine and granulocyte counts at each time point (weeks 1, 2, 4) following cisplatin administration.

Study 2: One-way ANOVA with Tukey's post hoc comparison was used to compare percentage change from baseline in plasma creatinine and granulocyte counts firstly, within each period and secondly, for each time point (weeks 1, 2, 3 and 4) for all periods of the study.

\section{Results}

Study 1: Comparison of the clinical dose and low dose of cisplatin

No vomits were recorded for dogs treated with placebo in either period 1 or period 2. Table 1 summarizes the characteristic of the emetic response for to both doses of cisplatin (onset, duration and number of events). The number of vomits was significantly different for the 2 doses of cisplatin $(P=0.03)$ (Fig. 2c). The latency to the onset of emetic effects of cisplatin was longer in dogs treated with the lower dose. The majority of vomits occurred between 4 and $6 \mathrm{~h}$ following the low-dose cisplatin versus $2-4 \mathrm{~h}$ with the clinical $\left(70 \mathrm{mg} / \mathrm{m}^{2}\right)$ dose (Fig. $2 \mathrm{a}, \mathrm{b}$ ).

Nausea-like behavior VAS scores were significantly increased compared to placebo for both doses of cisplatin (Fig. 3a, b). Table 1 summarizes the onset, duration and the peak nausea-like behavior response for both doses of cisplatin. The latency to the onset of nausea-like behavior was increased, and the peak score decreased for the low dose of cisplatin compared with the clinical $\left(70 \mathrm{mg} / \mathrm{m}^{2}\right)$ dose group. There was a $50 \%$ reduction in the AUC of the low compared to the clinical dose of cisplatin, this was statistically significant $(P=0.03)$.

\section{Arginine vasopressin}

Plasma AVP in the placebo-treated groups had the mean concentration of $1.78 \pm 0.97$ and $0.79 \pm 0.10 \mathrm{pmol} / \mathrm{l}$ for periods 1 and 2, respectively. Plasma AVP was significantly increased compared with placebo for both doses of cisplatin (Fig. 4a, b). The onset, duration, peak time and concentration of AVP is summarized in Table 2. The timecourse of change in plasma AVP compared to that of emesis and nausea-like behavior have a similar profile; however, the onset of the increase in plasma AVP concentrations appears to lag approximately $15 \mathrm{~min}$ behind increases in nausea-like behavior scores. The $\mathrm{AUC}_{0-7 \mathrm{~h}}$ of the low dose of cisplatin was reduced by $90 \%$ when compared to the clinical dose; however, a statistical difference could not be demonstrated $(P=0.089)$.

\section{Cortisol}

Plasma cortisol in the placebo-treated groups had the mean concentrations of $78.94 \pm 11.77$ and $139.44 \pm 15.09$ for periods 1 and 2, respectively. Plasma cortisol concentrations were significantly increased compared to placebo for both the cisplatin dose groups (Fig. 5a,b). Table 2 summarizes the change in plasma cortisol for both doses of cisplatin. As seen with the vasopressin response, the profile of the timecourse of change in plasma cortisol mimics that of emesis and nausea-like behavior and the cortisol he onset lags $15 \mathrm{~min}$ behind increases in nausea-like behavior. There was a $40 \%$ reduction in the $\mathrm{AUC}_{0-7 \mathrm{~h}}$ for the low compared to the clinical dose of cisplatin, this was not statistically significant $(P=0.218)$.

\section{Clinical safety monitoring}

All dogs completed studies 1 and 2 with the exception of $1 \mathrm{dog}$, which was removed from the last period of study 2 . This female dog was withdrawn from the study due to the flaring of atopic dermatitis unrelated to cisplatin treatment (requiring the daily use of corticosteroids for treatment).

\section{Plasma creatinine}

Study 1: Plasma creatinine was significantly increased from baseline at only the 1-week time point for the clinical dose of cisplatin and at no time for the low dose of cisplatin. The 

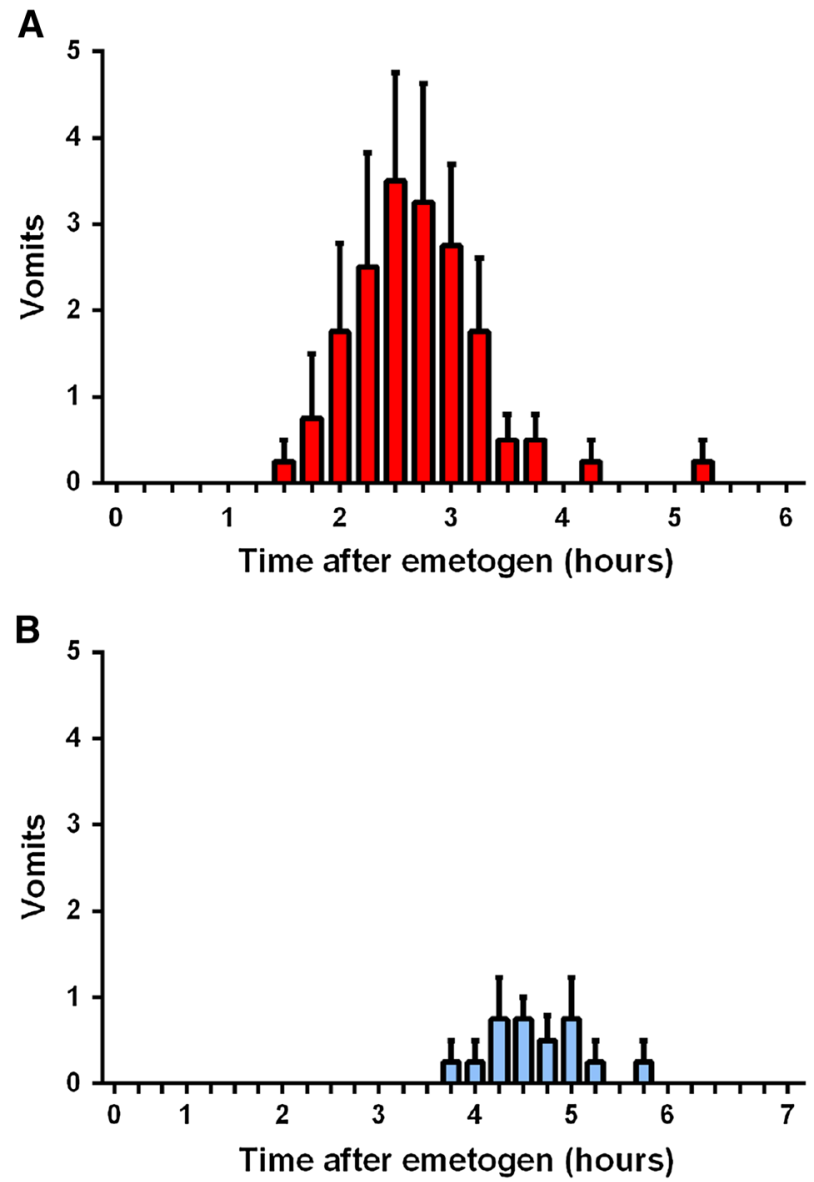

Fig. 2 Study 1 (clinical vs. low dose), vomits: timecourse of vomits following $70 \mathrm{mg} / \mathrm{m}^{2}$ cisplatin i.v. (a) or $15 \mathrm{mg} / \mathrm{m}^{2}$ cisplatin i.v. (b). Comparison of the mean number of vomits for the 70 and $15 \mathrm{mg} / \mathrm{m}^{2}$

plasma creatinine level at week 1 was significantly lower in the low dose compared to the clinical dose $(P=0.002)$. (Fig. 6a). Results were available only from 3 dogs for the clinical dose cisplatin at 1 week following cisplatin treatment. The plasma creatinine of one dog receiving the clinical cisplatin dose increased from baseline by $59 \mu \mathrm{mol} / \mathrm{l}$, the plasma creatinine of this dog subsequently normalized at week 2. Plasma creatinine did not increase by more than $20 \mu \mathrm{mol} / \mathrm{l}$ from baseline for all other dogs in both studies 1 and 2.

Study 2: Following repeated administration of cisplatin, no significant change in plasma creatinine was seen at any time point tested in each of the 4 periods of the study (Fig. 8a).

Granulocyte counts

Study 1: The granulocyte count at week 2 was significantly lower than the baseline $(P=0.005)$ following $70 \mathrm{mg} / \mathrm{m}^{2}$ cisplatin (Fig. 6b). Granulocyte count had recovered by

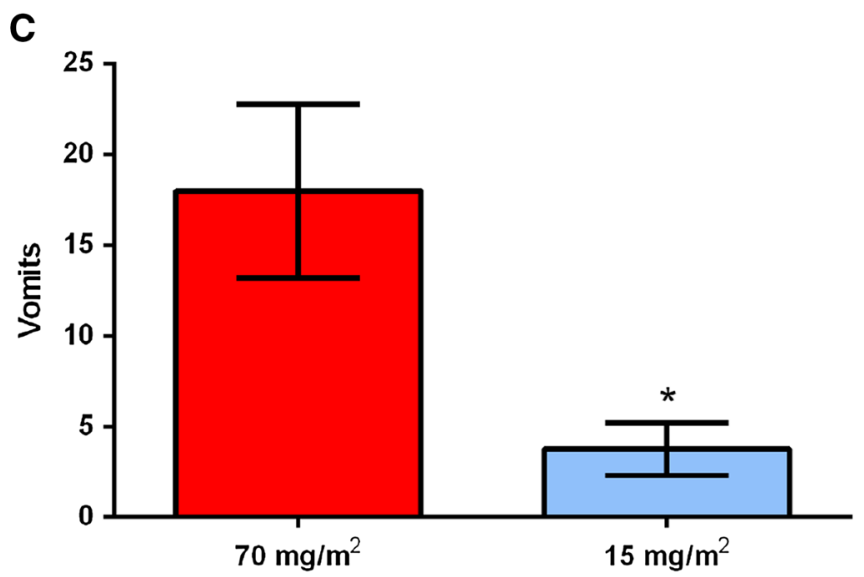

i.v. doses of cisplatin (c). Values expressed as mean \pm SEM, $n=4$ per dose group. $*$ Significant difference between cisplatin dose groups (Student's $t$ test $P=0.03$ )

week 4 . The decrease in granulocyte count at week 2 did not occur in group administered with the low dose of cisplatin $(P=0.002)$. No significant changes from baseline were observed for the low dose.

In the $70 \mathrm{mg} / \mathrm{m}^{2}$ dose group, 3 out of 4 dogs developed neutropenia, the number of granulocytes dropped below $2.0 \times 10^{3} / \mathrm{mm}^{3}$. Clinical signs of sepsis (lethargy, inappetence, pyrexia) were observed in 2 of the neutropenic dogs. The septic dogs received treatment with intravenous antibiotics (Amoxicillin and Clavulanic Acid; Augmentin, GSK, Middlesex, UK) and fluid therapy. Oral antibiotic treatment (Noroclav, Norbrook, Northhamptonshire, UK) was administered following the cessation of i.v. antibiotics and to the neutropenic dog that did not develop septic complications. Oral antibiotics were continued for a minimum of a week or until any clinical signs of sepsis had abated and granulocyte counts had exceeded $2.0 \times 10^{3} / \mathrm{mm}^{3}$.

Study 2: There was no significant difference between baseline granulocyte counts for periods $1-4$ of the study (Fig. 7). Granulocyte counts were compared to baseline for 

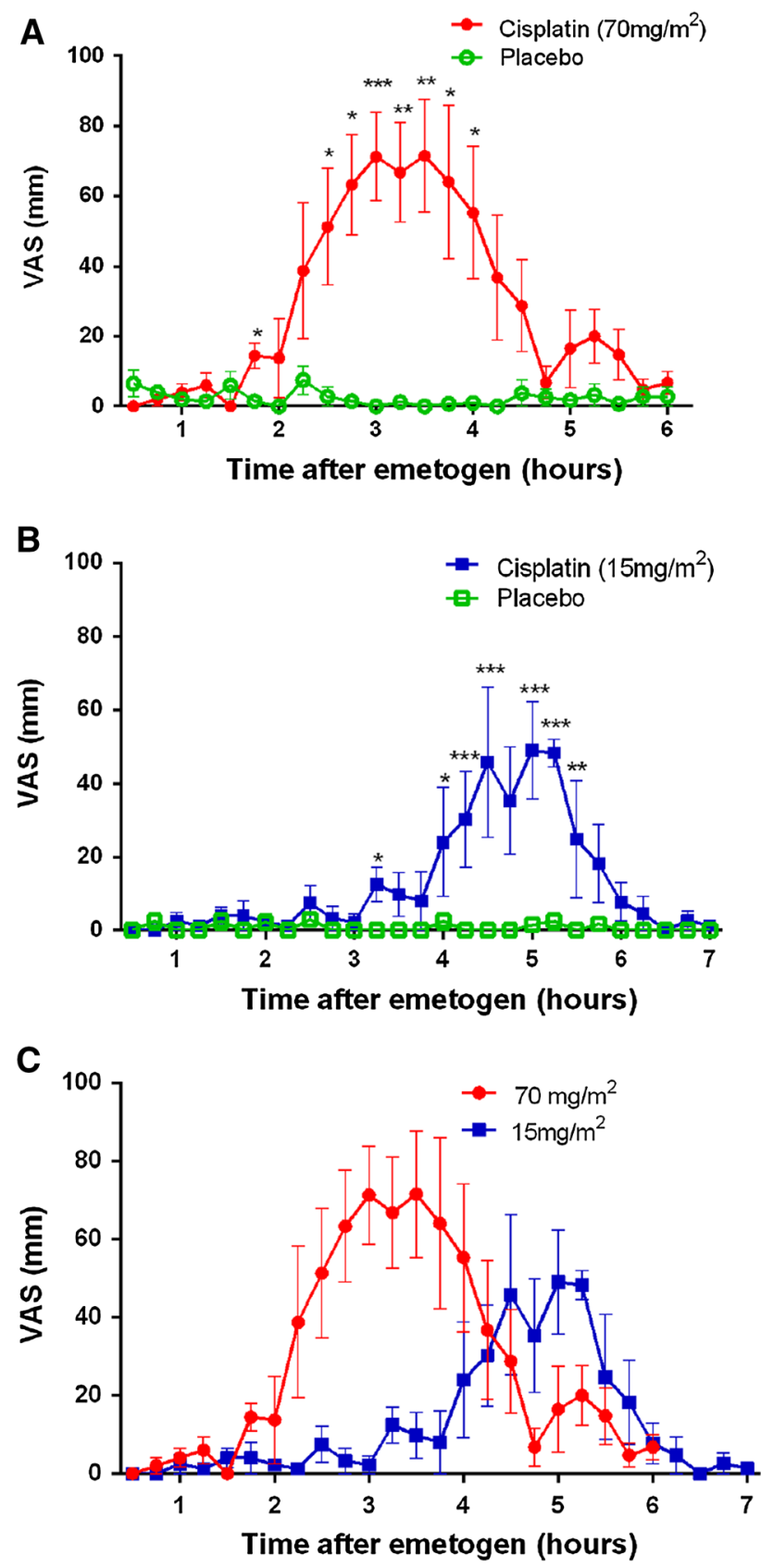

Fig. 3 Study 1 (clinical vs. low dose), nausea-like behavior: timecourse of nausea-like behavior (VAS) following $70 \mathrm{mg} / \mathrm{m}^{2}$ cisplatin i.v. compared to placebo (a) or $15 \mathrm{mg} / \mathrm{m}^{2}$ cisplatin i.v. compared to placebo (b). Comparison of the nausea-like behavior timecourse profiles for the 70 and $15 \mathrm{mg} / \mathrm{m}^{2}$ i.v. doses of cisplatin (c). Values presented as mean \pm SEM, $n=4$ per group. Significant differences between cisplatin- and placebo-treated groups (mixed linear model, $* P<0.05, * * P<0.01, * * * P<0.001)$

4 weeks following cisplatin administration in each period of the study (Fig. 8b). The only significant difference was observed in the 4th period of the study where relative change of granulocyte counts at week 3 was significantly lower than baseline following cisplatin administration
(Fig. 8c). Throughout the course of the study, the nadir of granulocytes occurred during week 3 of each period. The effect on granulocyte counts at 3 weeks seemed cumulative, worsening with further administrations of cisplatin. During the nadir at week 3,1 of 8 and 2 of 7 dogs became neutropenic periods 3 and 4 of the study, respectively. No clinical signs of sepsis were detected in any of the dogs that developed neutropenia in study 2 , but prophylactic antibiotics were given orally as described above for study 1 .

\section{Discussion}

The first goal of the present study was to refine the dose of cisplatin used to induce nausea-like behavior and emesis in the dog as a model to test the anti-nausea and anti-emetic properties of pharmacological agents.

The data presented for study 1 demonstrated that the low dose of cisplatin $\left(15 \mathrm{mg} / \mathrm{m}^{2}\right)$ was associated with an $80 \%$ reduction in the number of vomits making it a much more welfare friendly model in which to assess the efficacy of anti-nausea action of drugs. The dogs still demonstrated easily measurable signs of nausea-like behavior when compared to the placebo, which was $33 \%$ reduced compared to the clinical dose of cisplatin which has been traditionally used. The latency to the onset of vomiting and nausealike behavior was greater in the low-dose cisplatin group as occurs in the ferret where latency to onset is significantly greater in studies utilizing $5 \mathrm{mg} / \mathrm{kg}$ cisplatin compared to those using $10 \mathrm{mg} / \mathrm{kg}$ (Percie du Sert et al. 2011).

Another goal of cisplatin dose refinement was to determine whether repeated administration of the low dose was acceptable from a toxicity perspective to allow a crossover design. Three of the four dogs administered the clinical dose of cisplatin (75\%) demonstrated neutropenia at a level that made the risk of infection very high, with $50 \%$ of dogs showing clinical signs of sepsis after a single administration of cisplatin. This occurred after 2 weeks of cisplatin administration as would be expected based on the residual bone marrow reserve of white blood cells and the response time required for the regulation of neutrophil production (Dale et al. 1972). None of the dogs given the low dose of cisplatin showed neutropenia of this degree after a single dose. However, in study 2 where low-dose cisplatin was administered on four occasions separated by 28 days, a progressive reduction in granulocyte count at week 3 was noted. The neutropenic threshold below which the risk of infection is known to increase significantly was only breached in $25 \%$ of dogs receiving low-dose cisplatin after the fourth cycle and none of these dogs showed signs of sepsis. Ogilvie et al. (1988) reported a double nadir in granulocyte count at days 6 and 15 after administration of doses of $70 \mathrm{mg} / \mathrm{m}^{2}$ in healthy dogs. The nadirs we observed 


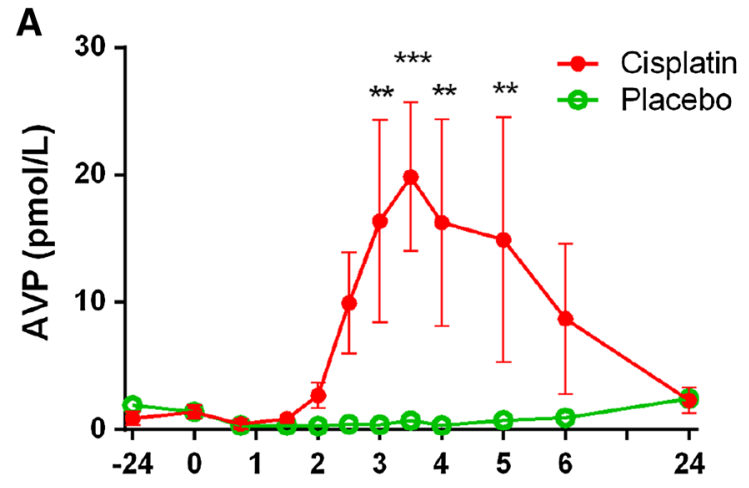

Fig. 4 Study 1 (high vs. low dose), arginine vasopressin: timecourse of change in plasma AVP following $70 \mathrm{mg} / \mathrm{m}^{2}$ cisplatin i.v. compared to placebo (a) or $15 \mathrm{mg} / \mathrm{m}^{2}$ cisplatin i.v. compared to placebo (b).

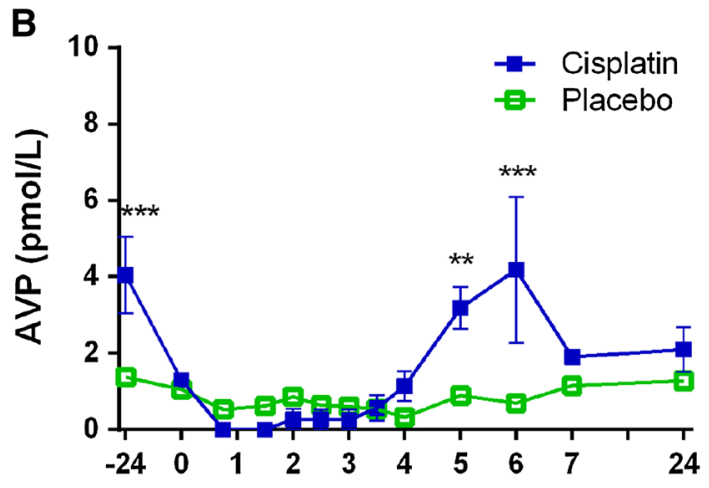

Values presented as mean \pm SEM, $n=4$ per group. Significant differences between cisplatin- and placebo-treated groups (mixed linear model, $* P<0.05, * * P<0.01, * * * P<0.001)$

Table 2 Summary of the biomarker responses following cisplatin administration

\begin{tabular}{|c|c|c|c|c|c|c|c|c|}
\hline \multirow[t]{2}{*}{ Dose } & \multicolumn{4}{|l|}{ Vasopressin } & \multicolumn{4}{|l|}{ Cortisol } \\
\hline & $\begin{array}{l}\text { Onset time } \\
\text { (h) }\end{array}$ & $\begin{array}{l}\text { Duration } \\
\text { (h) }\end{array}$ & $\begin{array}{l}\text { Peak time } \\
\text { (h) }\end{array}$ & $\begin{array}{l}\text { Peak concentration } \\
(\mathrm{pmol} / \mathrm{L})(\text { mean } \pm \mathrm{SEM})\end{array}$ & $\begin{array}{l}\text { Onset time } \\
(\mathrm{h})\end{array}$ & $\begin{array}{l}\text { Duration } \\
\text { (h) }\end{array}$ & $\begin{array}{l}\text { Peak time } \\
\text { (h) }\end{array}$ & $\begin{array}{l}\text { Peak concentration } \\
(\mathrm{nmol} / \mathrm{L})(\text { mean } \pm \mathrm{SEM})\end{array}$ \\
\hline $70 \mathrm{mg} / \mathrm{m}^{2}$ Period 1 & 2 & 4 & 3.5 & $19.8 \pm 5.8$ & 2 & 4 & 3.5 & $456 \pm 108.5$ \\
\hline $15 \mathrm{mg} / \mathrm{m}^{2}$ Period 2 & 4 & 3 & 6 & $4.2 \pm 1.9$ & 4 & 3 & 5 & $268 \pm 46.8$ \\
\hline
\end{tabular}

Onset of the increase in plasma vasopressin and cortisol was estimated by visual inspection of the data

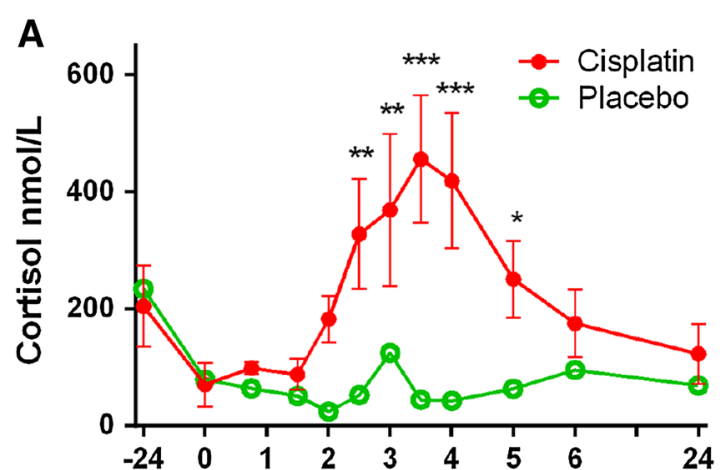

Fig. 5 Study 1 (clinical vs. low dose), cortisol: timecourse of change in plasma cortisol following $70 \mathrm{mg} / \mathrm{m}^{2}$ cisplatin i.v. compared to placebo (a) or $15 \mathrm{mg} / \mathrm{m}^{2}$ cisplatin i.v. compared to placebo (b). Values

were shifted to 7 and 21 days following administrations of the low dose cisplatin in study 2 .

Cisplatin also causes cumulative nephrotoxicity in clinical patients given $70 \mathrm{mg} / \mathrm{m}^{2}$ repeatedly (Ogilvie et al. 1992). In the present study, all dogs were fluid loaded and pretreated with mannitol to protect them from the nephrotoxicity of cisplatin. Serial plasma creatinine concentrations were used to detect acute kidney injury induced by cisplatin. This marker is not the most sensitive marker of acute kidney injury (AKI) as it is exponentially related to glomerular filtration rate (GFR), the gold standard for measuring

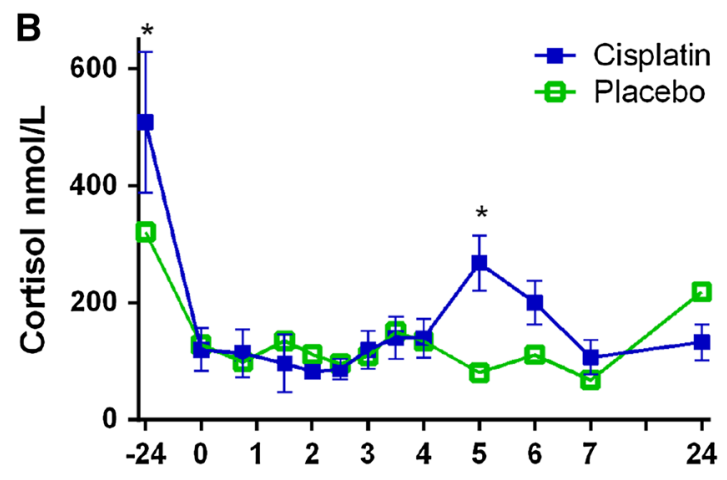

presented as mean \pm SEM, $n=4$ per group. Significant differences between cisplatin- and placebo-treated groups (mixed linear model, $* P<0.05, * * P<0.01, * * * P<0.001)$

functional renal mass (Loor et al. 2013). Plasma creatinine concentration changed significantly in dogs receiving the clinical dose of cisplatin at week 1 , and no significant increase in plasma creatinine concentration was determined over time in the dogs when they were given cisplatin repeatedly at the low dose. Ideally we would have measured GFR repeatedly following each dose of cisplatin administered. The technique for doing this was not compatible with the other procedures we wanted to conduct in the present study. Alternatively, we could have monitored urinary markers of renal damage in the present study. Unfortunately, these have 

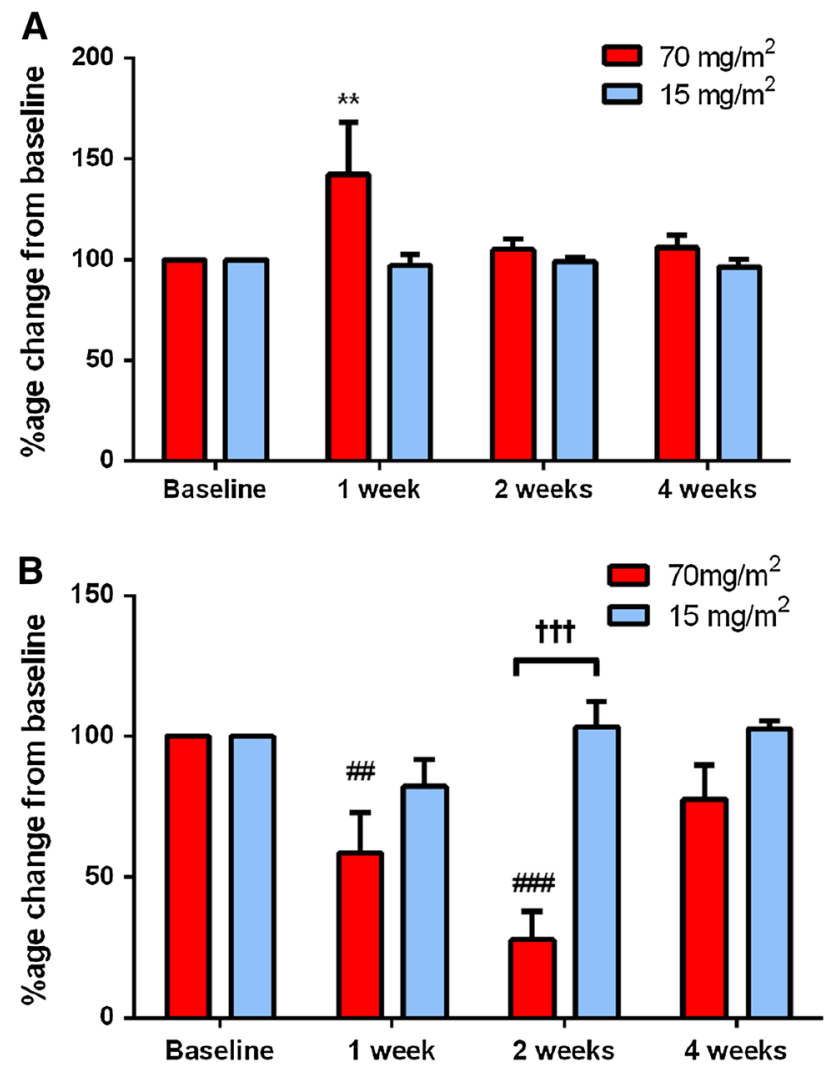

Fig. 6 Study 1 (clinical vs. low dose), clinical monitoring: Percentage change from baseline of plasma creatinine (a) and granulocytes (b) following cisplatin treatment with $70 \mathrm{mg} / \mathrm{m}^{2}$ in period 1 or $15 \mathrm{mg} /$ $\mathrm{m}^{2}$ in period 2. Values presented as mean \pm SEM, $n=4$ per group. *Significant difference between baseline and 1 week in the (univariate general linear model $P=0.001$ ). "Significant differences between baseline, 1 week and 2 weeks in the $70 \mathrm{mg} / \mathrm{m}^{2}$ cisplatin group (univariate general linear model $P=0.003 \& 0.000$, respectively). ${ }^{\dagger}$ Significant differences between cisplatin dose groups during week 2 (univariate general linear model $P=0.000$ )

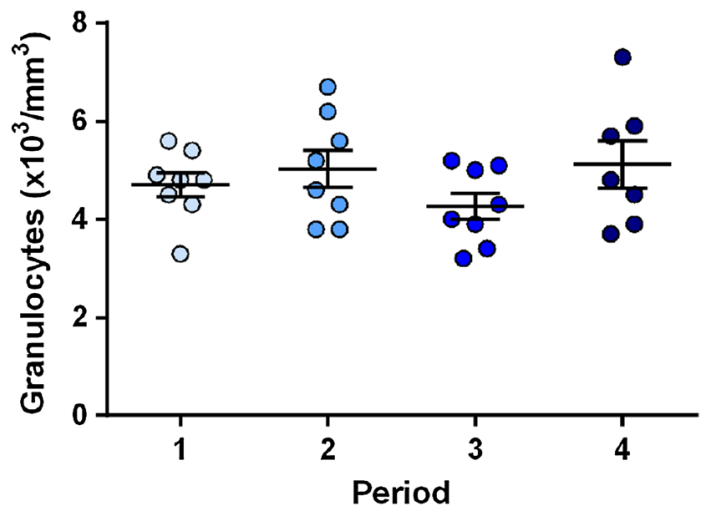

Fig. 7 Study 2 (4 periods repeated administration), clinical monitoring: comparison of baseline numbers of granulocytes at the baseline before each of the 4 periods of the study. Individual data presented with lines marking mean \pm SEM
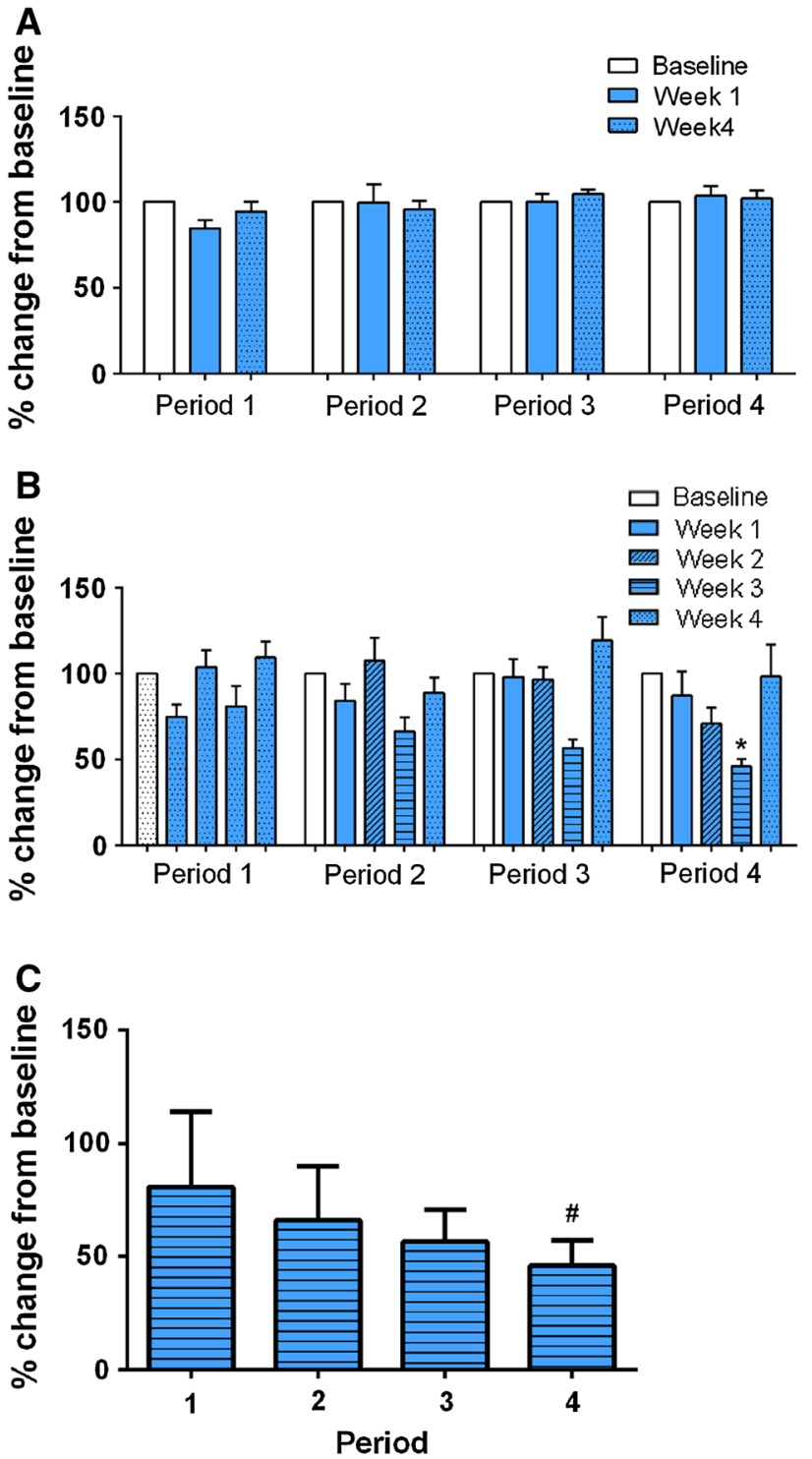

Fig. 8 Study 2 (4 periods repeated administration), clinical monitoring: percentage change from baseline of plasma creatinine (a) and granulocytes (b) following repeated administration of $18 \mathrm{mg} / \mathrm{m}^{2}$ cisplatin treatment in periods $1-4$ and comparison in the nadir granulocyte counts at 3 weeks for each period of study $2(\mathbf{c})$. *Significant difference between baseline and week 3 during period 4 (ANOVA, $P=0.017)$. "Significant difference in granulocyte counts at the 3 week time point between periods 1 and 4 of the study (ANOVA, $P=0.03)$

not been well characterized in the dog and the risk to the operators of collecting cisplatin-contaminated urine which would have been required to monitor changes in the urinary biomarkers over time was not considered to be justified.

Only one of the 4 dogs examined with the clinical dose of cisplatin showed an increase in plasma creatinine concentration that was of a magnitude that suggests AKI (>26.4 $\mu \mathrm{mol} / \mathrm{l}$ ) by the IRIS grading system (Cowgill 2013). None of the 8 dogs given the 4 doses of cisplatin at the 
low-dose rate demonstrated such an increase. This would suggest that AKI is not detectable from a single dose at the high-dose rate or with repeated administration of the lowdose rate. While accepting that the method used to detect AKI was not the most sensitive method and subclinical kidney injury could have occurred in both dosing regimens, it seems likely that the saline and mannitol infusions used in the present study afforded sufficient renoprotection to avoid significant renal damage from cisplatin. That this remained the case even when the drug is administered repeatedly at the lower refined dose rate, suggesting this repeated dosing regimen could be used in studies with a crossover design without significant risk of serious renal damage.

Overall, these findings suggest that cisplatin could be used safely at the lower refined dose rate thus facilitating studies of a crossover design. Furthermore, with repeated administration, the characteristic nausea-like behavioral response did not vary for the number of doses administered (data not shown), suggesting this model is not only safe but repeatable and suited to the testing of the effects of novel anti-nausea and anti-emetic compounds.

The development of a model that allows repeated administration better reflects the situation in human medicine where repeated doses of chemotherapeutic agents are administered for cancer treatment. Current animal models utilizing cisplatin produce a high frequency of emetic events. A metaanalysis of the literature reported $107 \pm 48$ retches and vomits in ferrets treated with $10 \mathrm{mg} / \mathrm{kg}^{-1}$ cisplatin during a 4-h observation period. A biphasic response to $5 \mathrm{mg} / \mathrm{m}^{2}$ cisplatin was reported producing $105 \pm 83$ and $340 \pm 171$ retches and vomits in the acute and delayed phases, respectively (Percie du Sert et al. 2011). Dogs experienced between 13 and 33 emetic events during the $6 \mathrm{~h}$ following administration of $70 \mathrm{mg} / \mathrm{m}^{2}$ cisplatin (de la Puente-Redondo et al. 2007). The extent of vomiting induced by cisplatin in these models is not indicative of the situation in the clinic where emesis is well controlled by modern anti-emetic combinations $\left(5-\mathrm{HT}_{3}\right.$ and $\mathrm{NK}_{1}$ antagonists) and nausea is now the most feared adverse effect of chemotherapy (de Boer-Dennert et al. 1997). For example, a complete response (no emesis and no rescue treatment) was achieved in $92 \%$ of patients for 5 days following cisplatin administration, but nausea was still reported by $53.8 \%$ of patients (Jang et al. 2013).

The second aim of this study was to identify putative biomarkers of nausea that might be used in drug development to highlight compounds with nauseogenic potential at the preclinical drug development phase in an objective way. While the results from this study are not sufficient to identify AVP and cortisol as biomarkers of nausea, they can be described as correlates due to the similarities between the AVP and cortisol responses compared to behavioral nausea.

AVP secretion from the posterior pituitary gland is classically regulated physiologically by two main stimuli, high plasma osmolality (initial defense mechanism against dehydration) and marked reduction in blood pressure (defense mechanism against severe hypotension threatening CNS arterial perfusion). The increases in AVP in dogs in study 1 at $\mathrm{t}_{-24 \mathrm{~h}}$ are likely to be associated with JVC placement and the related anesthesia and sedation, which occurred a few hours prior to the blood sample. AVP can be increased due to pain and surgical stress (Rose and Post 2001); however, this was a minimally invasive procedure. The nauseogenic effects of the sedative drugs (in particular alpha2 agonists) could provide the most likely explanation for the elevated AVP, which had returned to baseline noise level at the time of cisplatin administration. The significant increases in plasma AVP observed as a correlate of the behavioral response cannot be due to fluid imbalance from emesis as all dogs were fluid loaded with $0.9 \%$ saline solution prior to cisplatin administration and received fluid replacement throughout the course of the study in excess of fluid lost in vomits. In dogs deprived of water to stimulate AVP secretion, a pulsatile pattern of AVP release has been noted from baseline (van Vonderen et al. 2004). No evidence of pulsatile secretion was found in plasma AVP increases induced by cisplatin, for either dose, and is therefore different to the response caused by fluid loss or hyperosmolarity. This suggests once again that the AVP secretion associated with nausea-like behavior is not likely to be due to physiological responses associated with vomiting but could be related (directly or indirectly) to the nausea-like behavior, potential mechanisms discussed below.

The significant increase in plasma cortisol seen with both doses of cisplatin was unexpected. Human patients undergoing cancer chemotherapy treatment have decreased serum cortisol concentrations (Morrow et al. 2002). Additionally, patients with low urinary cortisol prior to chemotherapy treatment experience more severe nausea (Fredrikson et al. 1992). This is perhaps a species difference; however, cortisol was not repeatedly measured in the above studies to produce a timecourse of cortisol change following chemotherapy administration so there may be increases in cortisol in humans that have not yet been characterized.

A possible mechanism for the role of correlates, AVP and cortisol, in nausea is that AVP is released from the hypothalamus in response to emetic stimuli/stress to counteract potential water loss (Koch 1997). Increased AVP stimulates the release of adrenocorticotropic hormone (ACTH) from the anterior pituitary, which in turn stimulates cortisol release from the adrenal cortex (Chrousos and Gold 1998). The role of cortisol as a cause or effect of nausea is unclear. However, cortisol concentrations are controlled by a negative feedback system that includes the amygdala (Chrousos and Gold 1998), an area of the brain involved in nausea sensation (Napadow et al. 2013). 
The relationship of 'cause and effect' between AVP and nausea is unclear. Administration of exogenous AVP causes nausea (Caras et al. 1997; Kim et al. 1997). The present study shows the onset of nausea behavior precedes increases in biomarkers AVP and cortisol. This suggests, at least for cisplatin-induced nausea, that AVP release is an effect of the onset of nausea.

From the current study, it cannot be determined whether biomarkers AVP and cortisol are correlates of nausea-like behavior alone or nausea-like behavior and emesis. The AUCs of AVP and cortisol, while not statistically significant possibly due to small sample size, did exhibit a dose-dependent decrease for the 2 doses of cisplatin tested. The AUC of AVP displayed an $80 \%$ decrease from the clinical to the low dose of cisplatin; the AUC of cortisol was reduced by $40 \%$. This is not proportional to the significant $50 \%$ reduction in the nausea-like behavior AUC from the clinical to the low dose of cisplatin. The lack of proportionality in the changes in AUC would suggest that AVP and cortisol release is not directly dependent on the nausea-like behavior response. It is likely, especially in the case of AVP, that the emetic response is, at least partially, responsible for AVP release. To determine the suitability of AVP and cortisol as specific biomarkers of nausea, further experiment needs to be carried out ideally in a model of nausea which does not induce emesis. Due to the current lack of a specific nausea model, the current cisplatin-induced model of nausea and emesis could be used with the administration of anti-emetic treatment to block the emetic response while leaving the nausea response.

Two of the 3Rs are addressed by the low-dose cisplatin model of nausea and emesis. Firstly, 'refinement', the low dose still induces measurable nausea-like behavior and emesis while improving animal welfare by reducing the extreme number of vomits and the toxicity caused by the clinical dose. Secondly, 'reduction', reuse of the same cohort of animals in crossover studies designed with repeated administrations of cisplatin increases the statistical power of studies and reduces the number of dogs required. At the current time, 'replacement' is not a valid option for the study of nausea and emesis due to the complex physiology involved. Recently the ameba, dictyostelium, has been found to be able to identify emetic compounds through a change in chemotactic behavior (Robery et al. 2011). However, this response does not identify all emetogenic compounds; dictyostelium did not have a respond to apomorphine or any of the cytotoxic compounds screened including cisplatin. The ameba may be useful in the future for the replacement of animals in the initial screening of the emetic potential of non-cytotoxic compounds, but, at the end of the day, the ameba nor any other cell can vomit so animal models are still required. As nausea is a subjective sensation, sentience is essential for it to be experienced necessitating the use of animal models. Conditioned gaping is induced by the administration of emetogens to the rat (Parker and Limebeer 2006). This model has been useful in the elucidation of nausea neural pathways (Parker and Mechoulam 2003; Rock et al. 2008; Limebeer et al. 2010) in a common, cost-effective laboratory species. Ultimately, like cells, rats cannot vomit and the presence of nausea in a non-emetic species cannot be confirmed but this instead provides a model of a nausea-like response.

We recommend that the this model would be useful, not in the initial screening stages of compound testing, which could be carried out in more lower laboratory species such as the rat, but to determine the validity and translatability of nominated candidate compounds. The dog in particular is a good model for nausea and emesis in man as it is close to man in emetic sensitivity (Andrews et al. 1990; Percie du Sert et al. 2012), and therefore likely to have the greatest predictive validity of laboratory species. The unique nature of the human-canine bond has developed to allow humans to accurately predict the emotional state of the dog based on their facial expression (Bloom and Friedman 2013). This ability of dogs to communicate emotion to humans makes it a particularly relevant model for the study of a subjective sensation such as nausea. This communication was made more powerful in the present study by the steps taken by the investigators to acclimatize the dog to the experimental context and learn the individual characters of the dogs and how they respond to their environment. This led to an ability to detect and grade nausea-like behaviors in the dogs with a high level of sensitivity, which we contend would not be possible in species that do not bond with humans in the same way.

\section{Conclusion}

Subclinical doses of cisplatin $\left(15-18 \mathrm{mg} / \mathrm{m}^{2}\right)$ induce significant nausea-like behavior and emesis but with reduced toxicity. Increased safety achieved at these lower doses allows for crossover study designs, which include repeated administration of cisplatin in the same cohort of dogs. Plasma AVP and cortisol show potential as biomarkers of nausea; however, further studies are required to validate this finding fully.

Acknowledgments Financial support was provided by the Biotechnology and Biosciences Research Council (BBSRC). The authors would like to thank David Tattersall for his advice and guidance, YuMei Chang for her assistance with statistical tests also Frances Benstead, Abbe Martyn and Kate Thirgood for their excellent technical assistance.

Conflict of interest The authors have received financial support from Zoetis Ltd which produce Cerenia ${ }^{\mathrm{TM}}$ (maropitant citrate) an antiemetic for use in dogs and cats. Professor J Elliott has acted as a consultant for Zoetis Ltd. 


\section{References}

Andrews PL, Sanger GJ (2014) Nausea and the quest for the perfect anti-emetic. Eur J Pharmacol 722:108-121

Andrews PL, Rapeport WG, Sanger GJ (1988) Neuropharmacology of emesis induced by anti-cancer therapy. Trends Pharmacol Sci 9:334-341

Andrews PL, Davis CJ, Bingham S, Davidson HI, Hawthorn J, Maskell L (1990) The abdominal visceral innervation and the emetic reflex: pathways, pharmacology, and plasticity. Can J Physiol Pharmacol 68:325-345

Barabas K, Milner R, Lurie D, Adin C (2008) Cisplatin: a review of toxicities and therapeutic applications. Vet Comp Oncol 6:1-18. doi:10.1111/j.1476-5829.2007.00142.x

Bloom T, Friedman H (2013) Classifying dogs' (Canis familiaris) facial expressions from photographs. Behav Process 96:1-10

Caras SD, Soykan I, Beverly V, Lin Z, McCallum RW (1997) The effect of intravenous vasopressin on gastric myoelectrical activity in human subjects. Neurogastroenterol Motil 9:151-156

Chen JD, Qian L, Ouyang H, Yin J (2003) Gastric electrical stimulation with short pulses reduces vomiting but not dysrhythmias in dogs. Gastroenterology 124:401-409. doi:10.1053/g ast.2003.50048

Chrousos GP, Gold PW (1998) A healthy body in a healthy mindand vice versa-the damaging power of "uncontrollable" stress. J Clin Endocrinol Metab 83:1842-1845

Cowgill LD (2013) IRIS grading of acute kidney injury. International Renal Interest Society. http://www.iris-kidney.com/guidelines/ grading.shtml. Accessed 28 Apr 2014

Cubeddu LX (1992) Mechanisms by which cancer chemotherapeutic drugs induce emesis. Semin Oncol 19:2-13

Cubeddu LX, Lindley CM, Wetsel W, Carl PL, Negro-Vilar A (1990) Role of angiotensin II and vasopressin in cisplatin-induced emesis. Life Sci 46:699-705

Dale DC, Alling DW, Wolff SM (1972) Cyclic hematopoiesis: the mechanism of cyclic neutropenia in grey collie dogs. J Clin Invest 51:2197

de Boer-Dennert M, de Wit R, Schmitz PI, Djontono J, Beurden V, Stoter G, Verweij J (1997) Patient perceptions of the side-effects of chemotherapy: the influence of 5HT3 antagonists. Br J Cancer 76:1055-1061

de la Puente-Redondo VA, Tilt N, Rowan TG, Clemence RG (2007) Efficacy of maropitant for treatment and prevention of emesis caused by intravenous infusion of cisplatin in dogs. Am J Vet Res 68:48-56. doi:10.2460/ajvr.68.1.48

Fisher RD, Rentschler RE, Nelson JC, Godfrey TE, Wilbur DW (1982) Elevation of plasma antidiuretic hormones (ADH) associated with chemotherapy-induced emesis in man. Cancer Treat Rep 66:25-29

Fredrikson M, Hursti T, Furst CJ, Steineck G, Borjeson S, Wikblom M, Peterson C (1992) Nausea in cancer chemotherapy is inversely related to urinary cortisol excretion. Br J Cancer 65:779-780

Horn CC (2008) Why is the neurobiology of nausea and vomiting so important? Appetite 50:430-434

Imamura H, Ikeda M, Furukawa $\mathrm{H}$ et al (2006) Phase II study of protracted irinotecan infusion and a low-dose cisplatin for metastatic gastric cancer. World J Gastroenterol 12:6522

Ina S, Tani M, Kawai M et al (2008) Phase 2 trial of oral S-1 combined with low-dose cisplatin for unresectable advanced pancreatic cancer. Anticancer Res 28:2373-2377

Ito J, Okada M, Abo T, Okamoto Y, Yamashita T, Takahashi K (1987) Experimental study on the control of cisplatin-induced emesis in dogs. Gan To Kagaku Ryoho 14:706-710

Jang G, Song HH, Park KU et al (2013) A phase ii study to evaluate the efficacy of ramosetron, aprepitant, and dexamethasone in preventing cisplatin-induced nausea and vomiting in chemotherapy-naïve cancer patients. Cancer Res Treat 45:172-177

Kelland L (2007) The resurgence of platinum-based cancer chemotherapy. Nat Rev Cancer 7:573-584. doi:10.1038/nrc2167

Kim MS, Chey WD, Owyang C, Hasler WL (1997) Role of plasma vasopressin as a mediator of nausea and gastric slow wave dysrhythmias in motion sickness. Am J Physiol 272:G853-G862

Koch KL (1997) A noxious trio: nausea, gastric dysrhythmias and vasopressin. Neurogastroenterol Motil 9:141-142

Limebeer CL, Vemuri VK, Bedard H, Lang ST, Ossenkopp KP, Makriyannis A, Parker LA (2010) Inverse agonism of cannabinoid $\mathrm{CB} 1$ receptors potentiates $\mathrm{LiCl}$-induced nausea in the conditioned gaping model in rats. Br J Pharmacol 161:336-349. doi:10.1111/j.1476-5381.2010.00885.x

Loor J, Daminet S, Smets P, Maddens B, Meyer E (2013) Urinary biomarkers for acute kidney injury in dogs. J Vet Intern Med 27:998-1010

Minami M, Endo T, Hirafuji M et al (2003) Pharmacological aspects of anticancer drug-induced emesis with emphasis on serotonin release and vagal nerve activity. Pharmacol Ther 99:149-165

Morrow GR, Hickok JT, Andrews PL, Stern RM (2002) Reduction in serum cortisol after platinum based chemotherapy for cancer: a role for the HPA axis in treatment-related nausea? Psychophysiology 39:491-495

Napadow V, Sheehan JD, Kim J et al (2013) The brain circuitry underlying the temporal evolution of nausea in humans. Cereb Cortex 23:806-813. doi:10.1093/cercor/bhs073

Ogilvie GK, Krawiec DR, Gelberg HB, Twardock AR, Reschke RW, Richardson BC (1988) Evaluation of a short-term saline diuresis protocol for the administration of cisplatin. Am $\mathrm{J}$ Vet Res 49:1076-1078

Ogilvie GK, Fettman MJ, Jameson VJ et al (1992) Evaluation of a one-hour saline diuresis protocol for administration of cisplatin to dogs. Am J Vet Res 53:1666-1669

Olsson K, Bergstrom A, Kindahl H, Lagerstedt AS (2003) Increased plasma concentrations of vasopressin, oxytocin, cortisol and the prostaglandin F2alpha metabolite during labour in the dog. Acta Physiol Scand 179:281-287

Parker LA, Limebeer CL (2006) Conditioned gaping in rats: a selective measure of nausea. Autonomic Neurosci 129:36-41

Parker LA, Mechoulam R (2003) Cannabinoid agonists and antagonists modulate lithium-induced conditioned gaping in rats. Integr Physiol Behav Sci 38:133-145

Percie du Sert N, Rudd J, Apfel C, Andrews P (2011) Cisplatininduced emesis: systematic review and meta-analysis of the ferret model and the effects of 5-HT3 receptor antagonists. Cancer Chemother Pharmacol 67:667-686

Percie du Sert N, Holmes AM, Wallis R, Andrews PL (2012) Predicting the emetic liability of novel chemical entities: a comparative study. Br J Pharmacol 165:1848-1867. doi:10.1111/j.1476-5381.2011.01669.x

Portillo E, Mackin A, Hendrix P, Boyle C, Chrestman L (2006) Comparison of the modified Seldinger and through-the-needle jugular catheter placement techniques in the dog. J Vet Emerg Crit Care 16:88-95

Ramsey I (ed) (2012) BSAVA Small Animal Formulary. British Small Animal Veterinary Association, Gloucester

Robery S, Mukanowa J, Percie du Sert N, Andrews PL, Williams RS (2011) Investigating the effect of emetic compounds on chemotaxis in Dictyostelium identifies a non-sentient model for bitter and hot tastant research. PLoS ONE 6:e24439. doi:10.1371/ journal.pone.0024439

Rock EM, Limebeer CL, Mechoulam R, Piomelli D, Parker LA (2008) The effect of cannabidiol and URB597 on conditioned gaping (a model of nausea) elicited by a lithium-paired context 
in the rat. Psychopharmacology 196:389-395. doi:10.1007/ s00213-007-0970-1

Rose BD, Post TW (2001) Clinical physiology of acid-base and electrolyte disorders. McGraw-Hill, New York

Russell WMS, Burch RL, Hume CW (1959) The principles of humane experimental technique. Methuen \& Co. Ltd, London

Samson MK, Rivkin SE, Jones SE et al (1984) Dose-response and dose-survival advantage for high versus low-dose cisplatin combined with vinblastine and bleomycin in disseminated testicular cancer a southwest oncology group study. Cancer 53:1029-1035

Tidholm A, Haggstrom J, Hansson K (2005) Vasopressin, cortisol, and catecholamine concentrations in dogs with dilated cardiomyopathy. Am J Vet Res 66:1709-1717
Vail DM (2009) Supporting the veterinary cancer patient on chemotherapy: neutropenia and gastrointestinal toxicity. Top Companion Anim Med 24:122-129

van Vonderen IK, Wolfswinkel J, Oosterlaken-Dijksterhuis MA, Rijnberk A, Kooistra HS (2004) Pulsatile secretion pattern of vasopressin under basal conditions, after water deprivation, and during osmotic stimulation in dogs. Domest Anim Endocrinol 27:1-12. doi:10.1016/j.domaniend.2004.01.007

Yu X, Yang J, Hou X, Zhang K, Qian W, Chen JD (2009) Cisplatininduced gastric dysrhythmia and emesis in dogs and possible role of gastric electrical stimulation. Dig Dis Sci 54:922-927. doi:10.1007/s10620-008-0470-0 